\title{
Geometrical methods in hydrodynamics
}

\author{
Adrian Constantin
}

\begin{abstract}
We describe some recent results on a specific nonlinear hydrodynamical problem where the geometric approach gives insight into a variety of aspects.
\end{abstract}

\section{Introduction}

The geometric approach to hydrodynamical problems, initiated by Arnold [1], has the appealing feature that it represents the Lagrangian formulation of the mechanical problem. There is an extensive literature on geometric fluid mechanics (see the survey [3]) but the theory remains somewhat unsatisfactory. The description of ideal fluid flows by geometrical means consists mainly in formulating facts for the infinite-dimensional configuration space of fluid flows using established results from the finite-dimensional situation of classical Riemannian geometry cf. [3]. To circumvent the serious analytical difficulties encountered in working rigorously with the actual configuration space, Ebin and Marsden [13] enlarged this configuration space to spaces with a more convenient structure where a rigorous study can be pursued. Their work was further developed and prompted a variety of papers on the subject. Some significant recent contributions were made by Brenier [5] and Shnirelman [24] who introduced and studied generalized flows. However, the passage from results in weakened spaces of fluid configurations to the actual configuration space of the physical model remains an open question. Our aim is to describe ${ }^{1}$ the rigorous study of a specific hydrodynamical problem in configuration space. In doing so we hope to bring out several points of interest supporting the claim that certain hydrodynamical problems can be addressed through elegant geometrical methods. The obtained mathematical results have direct physical interpretations and illustrate the usefulness of the geometrical approach. This seems to be the first example where the geometric approach is put on a rigorous mathematical foundation but it is hoped that the present techniques will prove useful in a wider range of physical problems.

We consider a model for the unidirectional propagation of periodic shallow water waves - the Camassa-Holm model [6], a re-expression of the geodesic flow on the

\footnotetext{
${ }^{1}$ Throughout the presentation we give only an outline of the main ideas and refer to the appropriate papers for the full details.
} 
group of diffeomorphisms of the circle with the Riemannian structure induced by the $H^{1}$ right-invariant metric [22]. The core of the presentation is the rigorous geometric approach outlined in Section 3, approach that proves to be very useful in the qualitative study of the model. We discuss the question whether two arbitrary nearby fluid configurations can always be successive states of the flow cf. [9] and we describe the impact of the geometrical approach from [9] on the problem of the existence of local solutions. Section 2 is of a different nature and is not a prerequisite for Section 3. Its aim is to highlight the usefulness of geometrical considerations in making approximate models for physical processes: we discuss a derivation (alternative to the original derivation [6]) of the model from the governing equations for the water-wave problem by a variational approach cf. [7].

\section{A shallow water model}

\subsection{The governing equations for the water-wave problem}

Consider the propagation of a periodic plane progressive wave of small amplitude in the $x$-direction over water. The water surface is stationary in its undisturbed state $^{2}$ and the effects of surface tension are ignored. Let $(u(t, x, z), v(t, x, z))$ be the velocity of the water - no motion takes place in the $y$-direction so that there is only one horizontal component (in the direction of propagation) and the vertical component of the velocity field to be taken into account. The periodicity assumption means a periodic dependence in the $x$-variable (of, say, period one) of the velocity field. Let $z=h_{0}+\eta(t, x)$ be the water's free surface. The exact equations of motion for the velocity field are the equation of mass conservation

$$
\frac{\partial u}{\partial x}+\frac{\partial v}{\partial z}=0
$$

and Euler's equation of motion

$$
\left\{\begin{array}{l}
\frac{D u}{D t}=-\frac{1}{\rho} P_{x}, \\
\frac{D v}{D t}=-\frac{1}{\rho} P_{z}-g,
\end{array}\right.
$$

where $P(t, x, z)$ denotes the pressure, $g$ is the gravitational acceleration constant, $\rho$ is the (constant) density, and $D / D t$ is the material time derivative, $\frac{D f}{D t}=\frac{\partial f}{\partial t}+$ $u \frac{\partial f}{\partial x}+v \frac{\partial f}{\partial z}$. The boundary conditions are the dynamic boundary condition

$$
P=p_{0} \quad \text { on } \quad z=h_{0}+\eta(t, x)
$$

$p_{0}$ being the constant atmospheric pressure, decoupling the motion of the air from that of the water, together with the kinematic boundary conditions

$$
v=\eta_{t}+u \eta_{x} \quad \text { on } \quad z=h_{0}+\eta(t, x)
$$

and

$$
v=0 \quad \text { on } \quad z=0 .
$$

\footnotetext{
${ }^{2}$ The wave being created by, say, the action of the wind blowing over the still water surface, $z=0$ being the flat bottom and $z=h_{0}$ being the undisturbed water surface for a constant $h_{0}>0$.
} 
expressing the fact that the same particles always form the free water surface, i.e. $\frac{D}{D t}\left(z-h_{0}-\eta\right)=0$, respectively the fact that there is no flow normal to the horizontal bed. The general description of the propagation of a plane progressive wave is encompassed by the equations (2.1)-(2.5) cf. [17].

It is convenient to express (2.1)-(2.5) in terms of some fundamental parameters appearing after scaling the nondimensional form of the problem. Introducing a typical wavelength $L$ and a typical amplitude of the wave $a$, the set of nondimensional variables is

$$
x \mapsto L x, \quad z \mapsto h_{0} z, \quad t \mapsto \frac{L}{\sqrt{g h_{0}}} t, \quad u \mapsto u \sqrt{g h_{0}}, \quad v \mapsto v h_{0} \sqrt{g h_{0}} / L, \quad \eta \mapsto a \eta
$$

$x$ is replaced by $L x$, so that afterwards the symbol $x$ stands for a nondimensional variable etc.

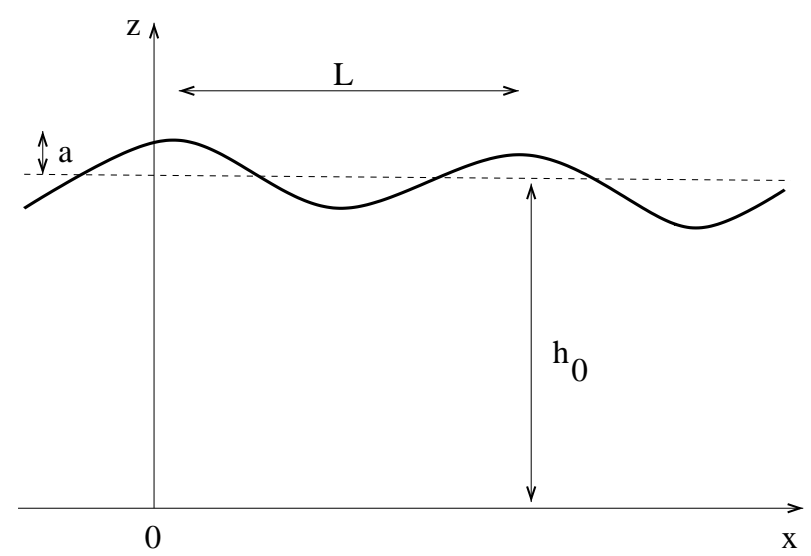

The nondimensional pressure variable $p$ measures the deviation from the hydrostatic pressure distribution $p_{0}+\rho g h_{0}(1-z)$ for a nondimensionalized $z$ (the hydrostatic pressure occurs if the water is stationary i.e. $u \equiv v \equiv 0)$,

$$
P=p_{0}+\rho g h_{0}(1-z)+\rho g h_{0} p .
$$

The problem (2.1)-(2.5) becomes

$$
\begin{cases}u_{t}+u u_{x}+v u_{z}=-p_{x}, & \\ \delta^{2}\left(v_{t}+u v_{x}+v v_{z}\right)=-p_{z}, & \\ u_{x}+v_{z}=0, & \text { on } z=1+\varepsilon \eta \\ p=\varepsilon \eta & \text { on } z=1+\varepsilon \eta \\ v=\varepsilon\left(\eta_{t}+u \eta_{x}\right) & \text { on } z=0 \\ v=0 & \text { r }\end{cases}
$$

in nondimensional variables, where $\varepsilon=a / h_{0}$ is the amplitude parameter and $\delta=$ $h_{0} / L$ is the shallowness parameter. Note that both $v$ and $p$, if evaluated on $z=$ $1+\varepsilon \eta$, are essentially proportional to $\varepsilon$. This observation is consistent with the fact that as $\varepsilon \rightarrow 0$ we must have $v \rightarrow 0$ and $p \rightarrow 0$ (with no disturbance the free surface becomes a horizontal surface on which $v=p=0$ ) and leads to the scaling of the nondimensional variables

$$
p \mapsto \varepsilon p, \quad(u, v) \mapsto \varepsilon(u, v) .
$$


Avoiding the introduction of a new notation, the problem (2.6) becomes

$$
\begin{cases}u_{t}+\varepsilon\left(u u_{x}+v u_{z}\right)=-p_{x}, & \\ \delta^{2}\left\{v_{t}+\varepsilon\left(u v_{x}+v v_{z}\right)\right\}=-p_{z}, & \\ u_{x}+v_{z}=0, & \text { on } z=1+\varepsilon \eta \\ p=\eta & \text { on } z=1+\varepsilon \eta \\ v=\eta_{t}+\varepsilon u \eta_{x} & \text { on } z=0 \\ v=0 & \end{cases}
$$

In respect of the well-posedness for the initial-value problem for (2.7) there has been significant recent progress [25] but some degree of mathematical intractability seems inevitable if one seeks a rigorous theory yielding an explanation of aspects of water waves that have been uncovered experimentally (e.g. wave interaction, wave breaking) by working with the governing equations (2.1)-(2.5).

\subsection{Derivation of the shallow water model}

The main impetus for a theory of shallow water waves that are periodic comes from the observation that, as a matter of common experience, the waves observed in a channel are approximately periodic.

A vast amount of theoretical work has been done to find schemes of approximation for replacing (2.1)-(2.5) with model equations that can be more effectively studied. The derivation of approximate models is based on formal perturbation schemes but, once a model equation is adopted, it is desirable to draw rigorous conclusions from it, confirmed as far as possible in experimental contexts. Water waves, which are considered a prime example of observability because they are easy to see and enjoy, are actually very difficult to be measured accurately in quantitative detail. This makes an answer to the question of preferability between alternative models very difficult ${ }^{3}$. At this point, let us recall that the virtue of the Lagrangian mechanics lies in the ease with which it can be used to formulate the equations of motion for complex mechanical problems. Lagrangian mechanics is based on a translation of the physical circumstances in abstract mathematical terms by describing motions of mechanical systems with no external forces as paths on a configuration space obtained from a variational principle ${ }^{4}$. Let us now describe how this approach leads to the derivation of an unidirectional shallow water model from the governing equations (2.1)-(2.5).

The shallow water limit corresponds cf. [17] to the condition $\delta \rightarrow 0$, so $p_{z}=$ $O\left(\delta^{2}\right)$ from the second equation in (2.6). Ignoring corrections of order $\delta^{2}$ we make the simplifying assumption that $p$ is independent of $z$. Then, from the first equation in (2.6) we infer that the horizontal acceleration $\frac{D u}{D t}=0$ for all particles in a plane $x=$ constant. Since the water was at rest in its undisturbed state, we find that

\footnotetext{
${ }^{3}$ However, a natural requirement should be that the model is amenable to a rigorous qualitative theory predicting properties of water waves that were established empirically.

${ }^{4}$ In Newtonian mechanics, the motion is the effect of certain forces while in Lagrangian mechanics the motion is a result of the attempt of Nature to minimize a functional - the physical laws of motion are replaced by a single postulate, the Action Principle.
} 
$u$ is the same for all particles in such a plane, so that $u$ is independent of $z$, i.e. $u=u(t, x)$. The third and last equation in (2.6) yield

$$
v=-z u_{x}
$$

that is, the vertical velocity of any particle is proportional to its height above the bottom. The approximation on the free surface for small $\varepsilon$, taking into account (2.8), is $u_{t}+\eta_{x}=0$ and $\eta_{t}+u_{x}=0$ (neglect $\varepsilon$ in the first and fourth relations from (2.7)). The general solution of this simple system is $\eta=u=F(x-t)$ where $F$ is any differentiable function, and thus

$$
\eta \approx u
$$

to a first approximation. By consistently neglecting the $\varepsilon$ contribution we will present derivation $^{5}$ of the Camassa-Holm equation from (2.7) by using variational methods in the Lagrangian formalism.

For a progressive plane wave no motion takes place in the $y$ direction and, as a particle on the surface will always stay on the surface, we may regard the motion as that of a one-dimensional compressible membrane ${ }^{6}$. $>$ From the Lagrangian viewpoint the motion of a mechanical system is described by a path of diffeomorphisms $\gamma(t, \cdot)$ of the ambient space - the knowledge of $\gamma(t, \cdot)$ giving the configuration of the particles at time $t$. The Lie group $\mathcal{D}$ of smooth orientation-preserving diffeomorphisms of the circle $\mathbb{S}$ represents the configuration space for the spatially periodic motion of one-dimensional mechanical systems. The material velocity field is $(t, x) \mapsto \gamma_{t}(t, x)$ while the spatial velocity field is given by $w(t, y)=\gamma_{t}(t, x)$ where $y=\gamma(t, x)$, i.e. $w(t, \cdot)=\gamma_{t} \circ \gamma^{-1}$. In terms of $w$ we have the Eulerian description (from the viewpoint of a fixed observer) while in terms of $\left(\gamma, \gamma_{t}\right)$ we have the Lagrangian description (the motion as seen from one of the particles). The velocity phase space is the tangent bundle $T \mathcal{D}$ of $\mathcal{D}$. Let $\mathcal{G}$ be the tangent space at the identity $I d$. The Lagrangian is a scalar function $\mathcal{L}: T \mathcal{D} \rightarrow \mathbb{R}$ and the action along a path $\{\gamma(t): 0 \leq t \leq T\}$ in $\mathcal{D}$ is defined as $\mathfrak{a}(\gamma)=\int_{0}^{T} \mathcal{L}\left(\gamma, \gamma_{t}\right) d t$. The Action Principle [2] states that the equation of motion is the equation satisfied by a critical point of the action in the space of paths $\{\gamma(t), 0 \leq t \leq T\}$ on $\mathcal{D}$, with fixed endpoints $\gamma(0)=\gamma_{0}$ and $\gamma(T)=\gamma_{1}$. Note the following right-invariance property: if we replace the path $t \mapsto \gamma(t)$ by $t \mapsto \gamma(t) \circ \gamma_{0}$ for a fixed time-independent $\gamma_{0} \in \mathcal{D}$, then the spatial velocity $w=\gamma_{t} \circ \gamma^{-1}$ is unchanged. This suggests to consider a right-invariant Lagrangian $\mathcal{L}: T \mathcal{D} \rightarrow \mathbb{R}$ by transporting a certain inner product $K: \mathcal{G} \rightarrow \mathbb{R}$ to all tangent spaces of $\mathcal{D}$ by means of right translations. The most natural choice for $K$ is the kinetic energy ${ }^{7}$. Taking into account (2.8), the kinetic

\footnotetext{
${ }^{5}$ We refer to [6] for the original derivation.

${ }^{6}$ Incompressibility in one dimension would mean linear motion. The incompressibility of the whole water body is expressed by (2.8).

${ }^{7}$ With this choice, for the case of a perfect fluid moving in a bounded smooth domain $M \subset$ $\mathbb{R}^{k}, k=2,3$, having the group of all volume-preserving diffeomorphisms of $M$ as configuration space, the critical point condition for the action is Euler's equation cf. [3]. A simple change of variables will confirm that the incompressibility condition is equivalent to the fact that the kinetic energy $\frac{1}{2} \int_{M}|V(t, \xi)|^{2} d \xi, V$ being the velocity field, is right-invariant.
} 
energy on the surface (over one period) is

$$
K=\frac{1}{2} \int_{\mathbb{S}}\left(u^{2}+v^{2}\right) d x=\frac{1}{2} \int_{\mathbb{S}}\left(u^{2}+(1+\varepsilon \eta)^{2} u_{x}^{2}\right) d x \approx \frac{1}{2} \int_{\mathbb{S}}\left(u^{2}+u_{x}^{2}\right) d x
$$

to the order of our approximation. Transforming $K$ to a right-invariant Lagrangian $\mathcal{L}$, the action on a path $\{\gamma(t): 0 \leq t \leq T\}$ on $\mathcal{D}$ is

$$
\mathfrak{a}(\gamma)=\frac{1}{2} \int_{0}^{T} \int_{\mathbb{S}}\left\{\left(\gamma_{t} \circ \gamma^{-1}\right)^{2}+\left[\partial_{x}\left(\gamma_{t} \circ \gamma^{-1}\right)\right]^{2}\right\} d x d t
$$

Let us now find the condition for a path $\gamma:[0, T] \rightarrow \mathcal{D}$, parametrized by arc length, to be a critical point of the action in the space of paths with fixed endpoints, i.e.

$$
\left.\frac{d}{d \epsilon} \mathfrak{a}(\gamma+\epsilon \alpha)\right|_{\epsilon=0}=0
$$

for every path $\alpha:[0, T] \rightarrow C^{\infty}(\mathbb{S})$ with endpoints at zero and such that $\gamma+\epsilon \alpha$ is a small variation of $\gamma$ on $\mathcal{D}$. A lengthy calculation (see [7]) yields

$$
\left.\frac{d}{d \epsilon} \mathfrak{a}(\gamma+\epsilon \alpha)\right|_{\epsilon=0}=-\int_{0}^{T} \int_{\mathbb{S}}\left(\alpha \circ \gamma^{-1}\right)\left[u_{t}-u_{t x x}+3 u u_{x}-2 u_{x} u_{x x}-u u_{x x x}\right] d x d t
$$

The resulting Euler-Lagrange equation is the Camassa-Holm equation

$$
u_{t}-u_{t x x}+3 u u_{x}=2 u_{x} u_{x x}+u u_{x x x}, \quad t>0, \quad x \in \mathbb{S} .
$$

where $u=\gamma_{t} \circ \gamma^{-1}$ and $t \mapsto \gamma(t) \in \mathcal{D}$ is the curve (parametrized by arc length) yielding the critical point of the action functional in the space of paths. In (2.10), $u(t, x)$ represents the horizontal velocity component of a unidirectional shallow water flow or, equivalently, the displacement of the free surface from the undisturbed (flat) state cf. (2.9). This is in agreement with the interpretation of $u(t, x)$ given originally in [6], where a different approximation procedure was adopted. To interpret $\gamma$, let us look at the movement of the free surface. As time increases, $t \mapsto \gamma(t, x)$ is the path followed by the fluid particle which is initially located at $x, \gamma_{t}(t, x)$ is the velocity of the particle at that instant, and $u(t, \gamma(t, x))$ is the flow velocity at the location $\gamma(t, x)$ at time $t$. For fixed $t$, the function $\gamma(t, \cdot)$ is an increasing diffeomorphism of $\mathbb{S}$, representing the rearrangement of the particles with respect to their initial positions.

\section{The geometric approach}

The Camassa-Holm equation (2.10) can also be obtained formally as the geodesic equation for the $H^{1}$ right-invariant metric ${ }^{8}$ on $\mathcal{D}$ cf. [22]. To describe the rigorous geometric derivation of (2.10), let us recall the definition of a "natural Lagrangian system" cf. [2]. If $G$, a Lie group, is the configuration space of a mechanical

${ }^{8} H^{k}(\mathbb{S}), k \in \mathbb{N}$, stands for the Sobolev space of $L^{2}(\mathbb{S})$-functions $f$ with distributional derivatives $\partial_{x}^{i} f$ up to order $k$ in $L^{2}(\mathbb{S})$, endowed with the norm $\|f\|_{H^{k}}^{2}=\sum_{i=0}^{k}\left\|\partial_{x}^{i} f\right\|_{L^{2}(\mathbb{S})}^{2}$. 
system, the tangent bundle $T G$ of $G$ is the velocity phase space of the system. For a nondegenerated inner product $\langle\cdot, \cdot\rangle$ on the Lie algebra $\mathcal{G}$ (the tangent space at the neutral element of the group $G$ ), the quantity $\frac{1}{2}\langle v, v\rangle, v \in \mathcal{G}$, is called the kinetic energy $K$. Extending $K$ by right-translation to a right-invariant Lagrangian $L: T G \rightarrow \mathbb{R}$, we define the action along a path $\{g(t): a \leq t \leq b\}$ in $G$ as $\int_{a}^{b} L\left(g, g_{t}\right) d t$. The Action Principle [2] states that the equation of motion is the equation satisfied by an extremal of the action in the space of curves on $G$ with fixed end conditions $g(a)=g_{0}$ and $g(b)=g_{1}$. We say that the Principle of Least Action holds if the paths described by the motion of a mechanical system are not only extremals but also (local) minimal values of the action functional cf. [2]. For the Principle of Least Action to hold, it is necessary that the equation of motion is the geodesic equation on the configuration manifold. Indeed, if $g(t), a \leq t \leq b$, is a $C^{1}$-regular path (i.e. $g_{t} \neq 0$ on $\left.[a, b]\right)$ joining $g(a)=g_{0}$ to $g(b)=g_{1}$, the action $\mathfrak{a}(g)=\frac{1}{2} \int_{a}^{b}\left\langle g_{t}, g_{t}\right\rangle d t$ depends on the parametrization of the path but the length $\mathfrak{l}(g)=\int_{a}^{b}\left\langle g_{t}, g_{t}\right\rangle^{\frac{1}{2}} d t$ (with respect to the defined right-invariant metric on $G$ ) does not depend on the parametrization and $\mathfrak{l}^{2}(g) \leq 2(b-a) \mathfrak{a}(g)$, with equality if and only if $\left\langle g_{t}, g_{t}\right\rangle$ is constant on $[a, b]$. Therefore the (local) minimum of the action is realized by the curve of minimal length joining $g_{0}$ to $g_{1}$.

The group $\mathcal{D}$ of smooth orientation-preserving diffeomorphisms of the circle $\mathbb{S}$ (the real numbers modulo 1), the composition of maps being the group operation, represents the configuration space for the spatially periodic motion of inertial onedimensional mechanical systems. $\mathcal{D}$ is a connected manifold modelled on the Fréchet space $C^{\infty}(\mathbb{S})$ of smooth maps of the circle (the family of real smooth maps on $\mathbb{R}$ of period one), cf. [15]. A sequence $u_{j} \rightarrow u$ in $C^{\infty}(\mathbb{S})$ if and only if $\left\|u_{j}-u\right\|_{H^{n}} \rightarrow 0$ as $j \rightarrow \infty$ for all the seminorms $\|\cdot\|_{H^{n}}, n \geq 0$. If $F_{1}, F_{2}$ are Fréchet spaces, $U \subset F_{1}$ is open and $f: U \subset F_{1} \rightarrow F_{2}$ is a continuous map, the derivative of $f$ at $u \in U$ in the direction $v \in F_{1}$ is defined by $D f(u) v=\lim _{t \rightarrow 0} \frac{f(u+t v)-f(u)}{t}$. We say that $f$ is $C^{1}$ on $U$ if the limit exists for all $u \in U, v \in F_{1}$, and if $D f: U \times F_{1} \rightarrow F_{2}$ is continuous cf. [15]. Higher derivatives are defined as derivatives of the lower ones. The composition and the inverse are both smooth maps from $\mathcal{D} \times \mathcal{D} \rightarrow \mathcal{D}$, respectively $\mathcal{D} \rightarrow \mathcal{D}$, so that the group $\mathcal{D}$ is a Lie group cf. [15], the bracket on its Lie algebra $T_{I d} \mathcal{D} \equiv C^{\infty}(\mathbb{S})$ being

$$
[u, v]=-\left(u_{x} v-u v_{x}\right), \quad u, v \in C^{\infty}(\mathbb{S})
$$

If $\mathcal{F}(\mathcal{D})$ is the ring of smooth real-valued functions defined on $\mathcal{D}$ and $\mathcal{X}(\mathcal{D})$ is the $\mathcal{F}(\mathcal{D})$-module of smooth vector fields on $\mathcal{D}$, we define for $X \in \mathcal{X}(\mathcal{D})$ and $f \in \mathcal{F}(\mathcal{D})$ the Lie derivative $\mathcal{L}_{X} f$ as

$$
\mathcal{L}_{X} f(\varphi)=\lim _{h \rightarrow 0} \frac{f(\varphi+h X(\varphi))-f(\varphi)}{h}, \quad \varphi \in \mathcal{D} .
$$

If $U \subset C^{\infty}(\mathbb{S})$ is open and $X, Y: U \rightarrow C^{\infty}(\mathbb{S})$ are smooth, we denote

$$
D_{X} Y(\varphi)=\lim _{h \rightarrow 0} \frac{Y(\varphi+h X(\varphi))-Y(\varphi)}{h}, \quad \varphi \in \mathcal{D} .
$$

The definition of the vector field $[X, Y]=D_{X} Y-D_{Y} X$ is covariant and gives globally the Lie bracket of $X, Y \in \mathcal{X}(\mathcal{D}), \mathcal{L}_{X} Y=[X, Y]$. Note that if $\mathcal{X}^{R}(\mathcal{D})$ 
is the space of all right-invariant smooth vector fields on $\mathcal{D}$, then $X \in \mathcal{X}^{R}(\mathcal{D})$ is determined by its value $u$ at $I d, X(\eta)=R_{\eta} u$ for $\eta \in \mathcal{D}$, where $R_{\eta}$ stands for the right translation. The bracket $[X, Y]$ of $X, Y \in \mathcal{X}^{R}(\mathcal{D})$ is a right-invariant vector field and $[X, Y](I d)=[u, v]$, where $u=X(I d), v=Y(I d)$, cf. [21].

A nondegenerate continuous inner product ${ }^{9}$ can be transported to each tangent space $T_{\eta} \mathcal{D}$ by right-translation, i.e.

$$
\langle V, W\rangle(\eta):=\left\langle V \circ \eta^{-1}, W \circ \eta^{-1}\right\rangle \quad \text { for } \quad V, W \in T_{\eta} \mathcal{D},
$$

and endows $\mathcal{D}$ with a right-invariant metric. In principle, we would expect the corresponding geodesic paths on $\mathcal{D}$ to represent the motion of a periodic onedimensional mechanical system satisfying the Least Action Principle. To realize this program one needs to extend methods that have been developed in classical (finite-dimensional) Riemannian geometry to the present infinite-dimensional setting. To define geodesics we need appropriate notions of parallel transport and covariant derivative. If conceptually the formal extension of the finite-dimensional definitions is rather straightforward, the success of the whole approach depends on whether the existence of geodesics can be proved and whether they are locally length-minimizing. We would like to point out some problems that have to be addressed: $\mathcal{D}$ is a Fréchet manifold so that the classical local existence theorem for differential equations with smooth right-hand side and the inverse function theorem do not necessarily hold [15]. Note that both are tools that are indispensable in classical Riemannian geometry ${ }^{10}$. Furthermore, the existence of a metric covariant derivative is not ensured on general grounds cf. [18] since we deal with a Fréchet manifold endowed with a weak metric (each open set of the topology induced by the inner product on $C^{\infty}(\mathbb{S})$ is open in $C^{\infty}(\mathbb{S})$ but the converse is not true).

A development related to the ideas considered in [1] yields an existence result for the covariant derivative which preserves a right-invariant metric on $\mathcal{D}$.

Theorem 1. [9] Assume that there exists a bilinear operator $B: C^{\infty}(\mathbb{S}) \times C^{\infty}(\mathbb{S}) \rightarrow$ $C^{\infty}(\mathbb{S})$ such that

$$
\langle B(u, v), w\rangle=\langle u,[v, w]\rangle, \quad u, v, w \in C^{\infty}(\mathbb{S}) .
$$

Then there exists a unique Riemannian connection on $\mathcal{D}$ associated to the rightinvariant metric $\langle\cdot, \cdot\rangle$. If $J \subset \mathbb{R}$ is an open interval, a $C^{2}$-curve $\varphi: J \rightarrow \mathcal{D}$ is a geodesic if and only if

$$
u_{t}=B(u, u), \quad t \in J
$$

where $u=\varphi_{t} \circ \varphi^{-1} \in T_{I d} \mathcal{D} \equiv C^{\infty}(\mathbb{S})$.

\footnotetext{
${ }^{9}$ That is, $u \mapsto\langle u, u\rangle$ is a continuous (hence smooth) map on $C^{\infty}(\mathbb{S})$ and the relation $\langle u, v\rangle=0$ for all $v \in C^{\infty}(\mathbb{S})$ forces $u=0$. Typical examples are the $H^{s}(\mathbb{S})$-inner products with $s \geq 0$.

${ }^{10} \mathrm{As}$ an example of the contrast with the finite-dimensional case (for the latter we refer to [23]), consider the Lie-group exponential map $v \rightarrow \exp _{L}(v) \in \mathcal{D}$, obtained by evaluating for each $v \in T_{I d} \mathcal{D}$ the flow $t \mapsto \eta(t, \cdot)$ determined by the differential equation

$$
\eta_{t}=v(\eta) \quad \text { in } \quad C^{\infty}(\mathbb{S})
$$

at $t=1$. The Lie-group exponential map is a smooth map of the Lie algebra to the Lie group but, although the derivative of $\exp _{L}$ at $0 \in C^{\infty}(\mathbb{S})$ is the identity, $\exp _{L}$ is not locally surjective cf. [21].
} 
Consider now the right-invariant metric on $\mathcal{D}$ defined by the $H^{1}$ inner product

$$
\langle u, v\rangle_{H^{1}}=\int_{\mathbb{S}}\left(u(x) v(x)+u^{\prime}(x) v^{\prime}(x)\right) d x, \quad u, v \in C^{\infty}(\mathbb{S}),
$$

on $T_{I d} \mathcal{D} \equiv C^{\infty}(\mathbb{S})$. A straightforward calculation yields

$$
B(u, v)=-\left(1-\partial_{x}^{2}\right)^{-1}\left(2 v_{x}\left(1-\partial_{x}^{2}\right) u+v\left(1-\partial_{x}^{2}\right) u_{x}\right), \quad u, v \in C^{\infty}(\mathbb{S}),
$$

so that Theorem 1 ensures the existence of a Riemannian connection. The geodesic equation for the $H^{1}$ right-invariant metric is the Camassa-Holm equation ${ }^{11}$

$$
u_{t}+u u_{x}+\partial_{x}\left(1-\partial_{x}^{2}\right)^{-1}\left(u^{2}+\frac{1}{2} u_{x}^{2}\right)=0
$$

where $t \mapsto \varphi(t, \cdot)$ is the geodesic curve starting at time $t=0$ at the identity $I d$ in the direction $u_{0} \in T_{I d} \mathcal{D}$ and $u=\varphi_{t} \circ \varphi^{-1}, \varphi_{t} \in T_{\varphi(t)} \mathcal{D}$. The geodesic equation is equivalent to the system

$$
\left\{\begin{array}{l}
\varphi_{t}=u(t, \varphi) \\
u_{t}+u u_{x}+\partial_{x}\left(1-\partial_{x}^{2}\right)^{-1}\left(u^{2}+\frac{1}{2} u_{x}^{2}\right)=0
\end{array}\right.
$$

with initial data $\varphi(0)=I d, u_{0} \in C^{\infty}(\mathbb{S})$. It is convenient to recast (3.5) as

$$
\left\{\begin{array}{l}
\varphi_{t}=v \\
v_{t}=P_{\varphi}(v)
\end{array}\right.
$$

where $v=u(t, \varphi)$ and the operator $P_{\varphi}$ is given by

$$
P_{\varphi}(v)=-\left\{\partial_{x}\left(1-\partial_{x}^{2}\right)^{-1}\left(\left(v \circ \varphi^{-1}\right)^{2}+\frac{1}{2}\left(v \circ \varphi^{-1}\right)_{x}^{2}\right)\right\} \circ \varphi .
$$

To show the existence of geodesics we adopt the following approach: we complete $C^{\infty}(\mathbb{S})$ under the $H^{k}(\mathbb{S})$-norm $(k \geq 3)$, deal with (3.6) on the resulting Hilbert manifold

$$
\mathcal{D}^{k}=\left\{\eta \in H^{k}(\mathbb{S}), \eta \text { is bijective, orientation preserving and } \eta^{-1} \in H^{k}(\mathbb{S})\right\},
$$

and then we show that the solutions of (3.6) are actually $C^{\infty}$ if the data is smooth. The map $(\varphi, v) \mapsto\left(v, P_{\varphi}(v)\right)$ is $C^{1}$ from a small neighborhood of $(I d, 0) \in \mathcal{D}^{k} \times H^{k}(\mathbb{S})$ to $H^{k}(\mathbb{S}) \times H^{k}(\mathbb{S})$ cf. [9]. Therefore, as a direct consequence of the fundamental theorem for ordinary differential equations on Hilbert manifolds, given an arbitrary $u_{0} \in H^{k}(\mathbb{S})$, the problem (3.5) has a unique solution $\left(\varphi, \varphi_{t}\right) \in C^{1}([0, T)) ; \mathcal{D}^{k} \times$ $\left.H^{k}(\mathbb{S})\right)$ for some $T>0$. This solution depends smoothly on the data. Since the composition regarded as a map $\mathcal{D}^{k+n} \times \mathcal{D}^{k} \rightarrow \mathcal{D}^{k}$ and the group inverse regarded as a map $\mathcal{D}^{k+n} \rightarrow \mathcal{D}^{k}$ are both of class $C^{n}$ if $k>3 / 2$ and $n \geq 0$ cf. [13], the relation $u=\varphi_{t} \circ \varphi^{-1}$ yields the following well-posedness result for the CamassaHolm equation

\footnotetext{
${ }^{11}$ Apply the operator $\left(1-\partial_{x}^{2}\right)$ to recover the form (2.10) derived in Section 2.2.
} 
Theorem 2. Let $k \geq 3$. Given $u_{0} \in H^{k}(\mathbb{S})$, the Camassa-Holm equation (2.10) has a unique solution $u \in C\left([0, T) ; H^{k}(\mathbb{S}) \cap C^{1}\left([0, T) ; H^{k-1}(\mathbb{S})\right.\right.$. Moreover, the solution depends continuously on the data.

Remark. The previous approach actually works if $k>3 / 2$, the only change being that for $k<3$ we replace (2.10) by the equivalent form (3.4). Theorem 2 was obtained with semigroup methods in [8] and it can be easily verified that the semigroup approach from [8] is valid also for $k>3 / 2$ (see also [19] for a different approach to well-posedness in $H^{k}(\mathbb{S})$ with $k>3 / 2$ ). Interestingly, the Camassa-Holm equation is not locally well-posed for $k<3 / 2$ cf. [16].

The existence of solutions to the geodesic equation on the enlarged configuration spaces being established ${ }^{12}$, the main question now is how to use this to deduce the existence of geodesics on $\mathcal{D}$. This is precisely the point where the rigorous approach usually breaks down and one can not deduce results on the actual configuration space (see the case of the Euler equation and of other hydrodynamical equations in [3]). However, in the case of the Camassa-Holm equation it is possible to deal with the actual configuration space. The essence of the matter is the following result ${ }^{13}$

Theorem 3. [9] Let $k \geq 3$. If $u$ is the solution of (3.4) with initial data $u_{0} \in H^{k}(\mathbb{S})$, defined for $t \in(-T, T)$, then

$$
m(t, \varphi(t, x)) \cdot \varphi_{x}^{2}(t, x)=m_{0}(x), \quad t \in(-T, T),
$$

for $m=u-u_{x x}$. Here $\varphi\left(t ; u_{0}\right) \in \mathcal{D}^{k}$ is the first component of the solution $(\varphi, u)$ of (3.5) with data $\left(I d, u_{0}\right)$.

A key point consists in the observation that all solutions of the Camassa-Holm equation with initial data $u_{0} \in H^{k}(\mathbb{S}), k \geq 3$, are uniformly bounded and the only way that a solution fails to exist for all time is that the wave breaks ${ }^{14}$ cf. [8]. Therefore the maximal existence time does not depend on the degree of smoothness of $u_{0} \in H^{k}(\mathbb{S}), k \geq 3$. This fact, combined with the continuous dependence on initial data of the solutions to (2.10), ensures that there is some $\delta>0$ so that all curves $t \mapsto \varphi\left(t ; u_{0}\right) \in \mathcal{D}^{k}$ are defined on [0,2], provided $\left\|u_{0}\right\|_{H^{3}}<\delta$.

Theorem 3 is instrumental in proving that if the initial data $u_{0} \in H^{k}(\mathbb{S}), k \geq 3$, does not belong to $H^{k+1}(\mathbb{S})$, then the corresponding curve $\varphi \in C^{2}\left([0, T) ; \mathcal{D}^{k}\right)$ is

\footnotetext{
${ }^{12}$ The Hilbert manifolds $\mathcal{D}^{k}$ are well-suited for analytic considerations e.g. for dealing with the existence of solutions to (3.5). However, it is important to point out that the $H^{1}$ right-invariant metric on $\mathcal{D}^{k}$ is not smooth and it has no covariant derivative on $\mathcal{D}^{k}$ cf. [9] so that their geometrical structure is deficient and they can serve only as tools.

${ }^{13}$ Theorem 3 is a consequence of the invariance of the metric by the action of the group on itself. More precisely, any $v \in C^{\infty}(\mathbb{S}) \equiv T_{I d} \mathcal{D}$ defines a one-parameter group of diffeomorphisms $h^{s}: \mathcal{D} \rightarrow \mathcal{D}, h^{s}(\varphi)=\varphi \circ \exp _{L}(s v)$, where $\exp _{L}$ is the Lie-group exponential map. Since the metric is by construction invariant under the action of $h^{s}$, it can be proved (see [9]) using Noether's theorem that$$
\int_{\mathbb{S}}\left(u-u_{x x}\right) \cdot \varphi_{x} \circ \varphi^{-1} \cdot v \circ \varphi^{-1} d x=\int_{\mathbb{S}}\left(u_{0}-u_{0, x x}\right) \cdot v d x .
$$

A change of variables and the arbitraryness of $v \in C^{\infty}(\mathbb{S})$ lead now to the invariance expressed by Theorem 3.

${ }^{14}$ This means that the solution remains bounded while its slope becomes unbounded at a finite time $T>0$.
} 
such that for all $t \in[0, T)$ we have $\varphi\left(t ; u_{0}\right) \notin \mathcal{D}^{k+1}$. We obtain the existence of geodesics on $\mathcal{D}$ - see [9]. Now we can define the Riemannian exponential map exp of the $H^{1}$ right-invariant metric, defined as the time-one map $\mathfrak{e x p}\left(u_{0}\right)=\varphi\left(1 ; u_{0}\right)$ for $\left\|u_{0}\right\|_{H^{3}}<\delta$. At this point, one proves that $\mathfrak{e x p}$ is a $C^{1}$ diffeomorphism from an open neighborhood $U_{3}$ of $0 \in H^{3}(\mathbb{S})$ to an open neighborhood $V_{3}$ of $I d$ on $\mathcal{D}^{3}$; we can take $U_{3}$ such that at every point of $U_{3}$, the differential of $\mathfrak{e x p}$ is a bijection of $H^{3}(\mathbb{S})$. Note that $U=U_{3} \cap C^{\infty}(\mathbb{S})$ and $V=V_{3} \cap C^{\infty}(\mathbb{S})$ are open neighborhhods of $0 \in C^{\infty}(\mathbb{S})$, respectively $I d \in \mathcal{D}$. The existence result for geodesics on $\mathcal{D}$ ensures that $\mathfrak{e x p}(U) \subset V$. On the other hand, we know from the previously mentioned result with regard to no gain of smoothness that if $\mathfrak{e x p}\left(u_{0}\right) \in V$ for some $u_{0} \in U_{3}$, then necessarily $u_{0} \in U$. Therefore $\mathfrak{e x p}$ is a local bijection from $U$ to $V$. Let $u_{0} \in U$. It is possible to show that $\mathfrak{e x p}$ is a $C^{1}$-map on every $U_{3} \cap H^{k}(\mathbb{S}), k \geq 3$, so that $D \mathfrak{e x p} u_{u_{0}}$ is a bounded linear operator from $H^{k}(\mathbb{S})$ to $H^{k}(\mathbb{S})$. At this point, Theorem 3 is crucial in proving inductively that $D \mathfrak{e x p} \mathfrak{p}_{u_{0}}$ is a bijection from $H^{k}(\mathbb{S})$ to $H^{k}(\mathbb{S})$ for all $k \geq 3$. Then, in view of the inverse function theorem for Hilbert manifolds, both $\mathfrak{e x p}$ and its inverse are $C^{1}$-maps on small $H^{k}(\mathbb{S})$-neighborhoods of $u_{0} \in U$, respectively $\mathfrak{e x p}\left(u_{0}\right) \in V$. But $k \geq 3$ is arbitrary so that $\mathfrak{e x p}$ is a $C^{1}$-diffeomorphism from $U$ to $V$. This is the main approach to the proof of

Theorem 4. [9] The Riemannian exponential map of the $H^{1}$ right-invariant metric on $\mathcal{D}$ is a $C^{1}$ diffeomorphism from a neighborhood of zero in $T_{I d} \mathcal{D} \equiv C^{\infty}(\mathbb{S})$ to a neighborhood of $I d$ on $\mathcal{D}$.

Pursuing the analysis, one can show that a geodesic is locally the shortest path between two closeby points of $\mathcal{D}$.

Theorem 5. [9] If $\eta, \varphi \in \mathcal{D}$ are close enough then $\eta$ and $\varphi$ can be joined by a unique geodesic. This unique geodesic is length minimizing among all piecewise $C^{1}$-curves joining $\eta$ to $\varphi$ on $\mathcal{D}$.

The physical interpretation of Theorem 5 is that a configuration of the system can be transformed to any nearby configuration by a unique flow of (2.10). Of all possible paths joining these two configurations, the system selects the one of minimal action.

Remark. If instead of the $H^{1}$ right-invariant metric on $\mathcal{D}$ we consider the $L^{2}$ rightinvariant metric, it turns out that the corresponding geodesic equation is the inviscid Burgers equation $u_{t}+3 u u_{x}=0$ cf. [3]. The existence of solutions to the geodesic equation on $\mathcal{D}$ can be proved but the Riemannian exponential map is not a $C^{1}$ local diffeomorphism cf. [9] and this shows that the geometric approach is not meaningful in this case.

\section{Appendix}

Let us highlight some of the properties of the Camassa-Holm model. Because this equation is relevant to water waves, we shall mainly review some properties that are peculiar to it in this respect. 
The prerequisite to the usefulness of any model equation for real wave phenomena is that solutions exist and are unique for a reasonable class of prescribed data. Also, the solutions should depend continuously on the data, as do the real phenomena that are simulated. The well-posedness issue is settled by Theorem 2 . We already pointed out in Section 3 that the only way that a solution for (2.10) fails to exist for all time is that the wave breaks cf. [8]. Moreover, the solution is defined for all times if and only if $y_{0}:=u_{0}-\partial_{x}^{2} u_{0}$ does not change properly sign cf. [20].

The discovery that the solitary waves of (2.10) are solitons $^{15}$ is due to Camassa and Holm [6]. We refer to [4] for a beautiful description of the soliton interaction. The solitons are stable (i.e. a wave starting close to a soliton will stay close to some translate of it and therefore will have approximately the same shape for all times) cf. [12], a fact that is relevant to the role of (2.10) as a meaningful physical approximation: the solitons (very special solutions) can be detected. The soliton aspect of the Camassa-Holm equation is related to the fact that the equation has infinitely many invariants being an integrable infinite-dimensional Hamiltonian system ${ }^{16}$.

\section{References}

[1] V. Arnold. Sur la géometrie différentielle des groupes de Lie de dimension infinie et ses applications à l'hydrodynamique des fluides parfaits. Ann. Inst. Fourier (Grenoble), 16:319-361, 1966.

[2] V. Arnold. Mathematical Methods of Classical Mechanics. Springer Verlag, New York, 1989.

[3] V. Arnold and B. Khesin. Topological Methods in Hydrodynamics. Springer Verlag, New York, 1998.

[4] R. Beals, D. Sattinger, and J. Szmigielski. Multipeakons and a theorem of Stieltjes. Inverse Problems, 15:1-4, 1999.

[5] Y. Brenier. Minimal geodesics on groups of volume-preserving maps and generalized solutions to the Euler equations. Comm. Pure Appl. Math., 52:411-452, 1999.

\footnotetext{
${ }^{15}$ Solitary waves - solutions of the form $u(t, x)=f(x-c t)$ which travel with fixed speed $c$ and vanish at infinity - which emerge completely unscathed upon collision are called solitons. It turns out that the Camassa-Holm solitons are peaked waves and have therefore to be understood as weak solutions. The local existence theory presented in Section 3 does not cover the case of the solitons; we refer to [11] for this.

${ }^{16}$ In the sense of the infinite-dimensional extension of a classical completely integrable Hamiltonian system: there is a transformation which converts the equation into an infinite sequence of linear ordinary differential equations which can be trivially integrated. The integrability of (2.10) was established formally in [6]. This issue was pursued rigorously in [10] where the direct and inverse spectral theory for (2.10) is clarified and expanded upon. The existence of an infinite number of conservation laws for (2.10) has been addressed previous to the derivation of the equation as a model for water waves in [14] where it was singled out as an abstract equation with some interesting properties.
} 
[6] R. Camassa and D. Holm. An integrable shallow water equation with peaked solitons. Phys. Rev. Letters, 71:1661-1664, 1993.

[7] A. Constantin. A Lagrangian approximation to the water-wave problem. Appl. Math. Lett., to appear.

[8] A. Constantin and J. Escher. Well-posedness, global existence, and blow-up phenomena for a periodic quasi-linear hyperbolic equation. Comm. Pure Appl. Math., 51:475-504, 1998.

[9] A. Constantin and B. Kolev. On the geometric approach to the motion of inertial mechanical systems. Technical Report 6, Lund University, 2001.

[10] A. Constantin and H. P. McKean. A shallow water equation on the circle. Comm. Pure Appl. Math., 52:949-982, 1999.

[11] A. Constantin and L. Molinet. Global weak solutions for a shallow water equation. Comm. Math. Phys., 211:45-61, 2000.

[12] A. Constantin and W. Strauss. Stability of peakons. Comm. Pure Appl. Math., 53:603-610, 2000.

[13] D. Ebin and J. E. Marsden. Groups of diffeomorphisms and the notion of an incompressible fluid. Ann. of Math., 92:102-163, 1970.

[14] A. S. Fokas and B. Fuchssteiner. Symplectic structures, their Bäcklund transformation and hereditary symmetries. Physica D, 4:47-66, 1981.

[15] R. Hamilton. The inverse function theorem of Nash and Moser. Bull. Amer. Math. Soc., 7:65-222, 1982.

[16] A. Himonas and G. Misiolek. The Cauchy problem for an integrable shallowwater equation. Differential and Integral Equations, 14:821-831, 2001.

[17] R. S. Johnson. A Modern Introduction to the Mathematical Theory of Water Waves. Cambridge University Press, 1997.

[18] S. Lang. Fundamentals of Differential Geometry. Springer Verlag, New York, 1999.

[19] Yi Li and P. Olver. Well-posedness and blow-up solutions for an integrable nonlinearly dispersive model wave equation. J. Differential Equations, 162:2763, 2000 .

[20] H. P. McKean. Breakdown of a shallow water equation. Asian J. Math., 2:203208, 1998.

[21] J. Milnor. Remarks on infinite-dimensional Lie groups. In Relativity, Groups and Topology (Les Houches, 1983), pages 1009-1057. North-Holland, Amsterdam, 1984. 
[22] G. Misiolek. A shallow water equation as a geodesic flow on the Bott-Virasoro group. J. Geom. Phys., 24:203-208, 1998.

[23] P. Olver. Applications of Lie Groups to Differential Equations. Springer Verlag, New York, 1993.

[24] A. Shnirelman. Generalized fluid flows, their approximation and applications. Geom. Funct. Anal., 4:586-620, 1994.

[25] S. Wu. Well-posedness in Sobolev spaces of the full water wave problem in 3-D. J. Amer. Math. Soc., 12:445-495, 1999.

Department of Mathematics,

Lund University, PO Box 118,

S-22100, Lund, Sweden.

adrian.constantin@math. lu.se 